\title{
Shared Hyperplanes and Normal Families of Holomorphic Curves
}

\author{
Xiaojie Zheng \\ College of Science, University of Shanghai for Science and Technology, Shanghai, China \\ Email: 1430974500@qq.com
}

How to cite this paper: Zheng, X.J. (2021) Shared Hyperplanes and Normal Families of Holomorphic Curves. American Journal of Computational Mathematics, 11, 83-93. https://doi.org/10.4236/ajcm.2021.112008

Received: March 24, 2021

Accepted: May 9, 2021

Published: May 12, 2021

Copyright $\odot 2021$ by author(s) and Scientific Research Publishing Inc. This work is licensed under the Creative Commons Attribution International License (CC BY 4.0).

http://creativecommons.org/licenses/by/4.0/

\begin{abstract}
In theorem LP [1], Liu proves the theorem when $N=2$, but it can't be extended to the general case in his proof. So we consider the condition that the families of holomorphic curves share eleven hyperplanes, and we get the theorem 1.1 .
\end{abstract}

\section{Keywords}

Hyperplane, Normal Family, Holomorphic Curve, Hyperplanes of Share

\section{Introduction}

Some theoretical and practical problems of research often boil down to the whole function and normality of meromorphic function. In 1907, P. Montel raised the concept of normal family. And nowadays, the theory of normal family has a good development. The core of the theory of normal family is the study of the format rule. It is possible to relate the normality of a family of the function and the function value for Nevanlinna value distribution theory in the 1930s. In 1975, Israel mathematician L.Z Alcman, starting from Marty formal rule, gave the new method of formal rule called Zalcman lemma. Then, in 1989, Pang Xuecheng improved Zalcman's way, who got the Pang-Zalcman lemma, and he made it possible to study involving the formal rule of derived function.

In high-dimensional complex manifold holomorphic mappings about formal rule research, Tu Zhenhan firstly studied normality problems which involves the multiplicity of holomorphic mappings in 1999. Then, in 2014, Yang Liu, Caiyun Fang and Xuecheng Pang who use the high-dimensional Zalcman lemma of GAladro and S.G Krantz, generalize two family of meromorphic function normal rule, which was proved by Xiao-jun Liu, Lisan Hua and Pang Xuecheng, and they get the relative results about the holomorphic curves. 
In value distribution and normal family theory of meromorphic functions, there is a well-known phenomenon named Bloch's heuristic principle, that is to say, a family of meromorphic functions which have a property $\mathcal{P}$ in common $D \subset \mathbb{C}$ should be normal on $D$ if this property $\mathcal{P}$ forces a meromorphic function on the whole complex plane $\mathbb{C}$ to be constant. Later, Zalcman (see [2] [3]) formulated a more precise statement (known as Zalcman's principle and Zalcman-Pang principle) to determine the normality for families of meromorphic functions.

In the case of holomorphic curves, there does still exist some similar phenomenon.

Firstly, Chen and Yan [4] proved the following result concerning uniqueness theorem for holomorphic curves.

Theorem CY. Let $f$ and $g$ be two nonconstant holomorphic curves from $\mathbb{C}$ to $\mathbb{P}^{N}(\mathbb{C})$, and $H_{j}, 1 \leq j \leq q$ be $q$ hyperplanes in $\mathbb{P}^{N}(\mathbb{C})$ located in general position such that $f(\mathbb{C}) \varsubsetneqq H_{j}, g(\mathbb{C}) \varsubsetneqq H_{j}$ and $f(z)$ "share" $H_{j}$ with $g(z)$, $1 \leq j \leq q$. If $q \geq 2 N+3$, then $f=g$.

Remark 1.1. Here, "share" means not only $f(z) \in H_{j} \Leftrightarrow g(z) \in H_{j}$, but also requires $f(z)=g(z)$ on those points where $f^{-1}\left(H_{j}\right)=g^{-1}\left(H_{j}\right)$.

Later, in [5], Yang et al. considered the corresponding result in normal family theory of holomorphic curves and obtained.

Theorem YFP. $\mathcal{F}$ is a family of holomorphic curves from a domain $D \subset \mathbb{C}$ into $\mathbb{P}^{N}(\mathbb{C})$ and $H_{j}, 1 \leq j \leq q$ be $q \geq 2 N+1$ hyperplanes in $\mathbb{P}^{N}(\mathbb{C})$ located in general position. Suppose that for each $f, g \in \mathcal{F}, f(z) \in H_{j} \Leftrightarrow g(z) \in H_{j}$, $z \in D, 1 \leq j \leq q$. Then $\mathcal{F}$ is normal on $D$.

Obviously, the condition in Theorem CY is much stronger than those in Theorem YFP. Naturally, a question is posed that how to narrow the gap.

Liu et al. [6] used a special curve of $\mathrm{f}$ named derived curve to replace $g$ and proved the following theorem.

Theorem LPY. Let $\mathcal{F}$ be a family of holomorphic maps of a domain $D \subset \mathbb{C}$ to $\mathbb{P}^{N}(\mathbb{C})$. Let $H_{l}=\left\{x \in \mathbb{P}^{N}(\mathbb{C}):\left\langle x, \alpha_{l}\right\rangle=0\right\}$ be hyperplanes in $\mathbb{P}^{N}(\mathbb{C})$ located in general position, where $\alpha_{l}=\left(a_{l 0}, \cdots, a_{l N}\right)^{\mathrm{T}}, a_{l 0} \neq 0, l=1,2, \cdots, 2 N+1$. Assume also that the following two conditions hold for every $f \in \mathcal{F}$ :

1) If $f(z) \in H_{l}$, then $\nabla f \in H_{l}, l=1,2, \cdots, 2 N+1$

2) If $f(z) \in \bigcup_{l=1}^{2 N+1} H_{l}$, then $\frac{\left|\left\langle f(z), H_{0}\right\rangle\right|}{\|f\|\left\|H_{0}\right\|} \geq \delta$, where $0<\delta<1$ is constant, $H_{0}=\left\{w_{0}=0\right\}$.

Then $\mathcal{F}$ is normal on $D$.

In [1], Liu et al. remove the restriction of the first coefficients of hyperplanes of above theorem in the case $\mathbb{P}^{2}(\mathbb{C})$ and prove the following theorem.

Theorem LP. $\mathcal{F}$ is a family of holomorphic maps of a domain $D \subset \mathbb{C}$ to $\mathbb{P}^{2}(\mathbb{C})$. Let $H_{0}=\left\{w_{0}=0\right\}, H_{l}=\left\{x \in \mathbb{P}^{2}(\mathbb{C}):\left\langle x, \alpha_{l}\right\rangle=0\right\} \neq H_{0}$ be hyperplanes in $\mathbb{P}^{2}(\mathbb{C})$ located in general position, where $\alpha_{l}=\left(a_{l 0}, a_{l 1}, a_{l 2}\right)^{\mathrm{T}}, l=1,2,3,4,5$. Assume the following conditions hold for every $f \in \mathcal{F}$ : 
1) $f(z) \in H_{l}$ if and only if $\nabla f \in H_{l}, l=1,2,3,4,5$

2) If $f(z) \in \bigcup_{l=1}^{5} H_{l}$, then $\frac{\left\langle f(z), H_{0}\right\rangle \mid}{\|f\|\left\|H_{0}\right\|} \geq \delta$, where $0<\delta<1$ is a constant.

Then $\mathcal{F}$ is normal on $D$.

Unfortunately, the proof this proof of this theorem cannot be generalized to the $\mathbb{P}^{N}(\mathbb{C})$, and we get the main result of article is as follows:

Theorem 1.1. Let $\mathcal{F}$ be a family of holomorphic maps of a domain $D \subset \mathbb{C}$ to $\mathbb{P}^{4}(\mathbb{C})$. Let $H_{0}=\left\{w_{0}=0\right\} \quad$ and $H_{l}=\left\{x \in \mathbb{P}^{4}(\mathbb{C}):\left\langle x, \alpha_{l}\right\rangle=0\right\} \neq H_{0}$ be hyperplanes in $\mathbb{P}^{4}(\mathbb{C})$ located in general position, where $\alpha_{l}=\left(\alpha_{l_{0}}, \alpha_{l_{1}}, \alpha_{l_{2}}, \alpha_{l_{3}}, \alpha_{l_{4}}\right)^{\mathrm{T}}, l=1,2,3, \cdots, 11$

Assume the following conditions hold for every $f \in \mathcal{F}$ :

1) $f(z) \in H_{l}$ if and only if $\nabla f \in H_{l}, l=1,2,3, \cdots, 11$

2) If $f(z) \in \bigcup_{l=1}^{11} H_{l}$, then $\frac{\left\langle f(z), H_{0}\right\rangle \mid}{\|f\|\left\|H_{0}\right\|} \geq \delta$, where $0<\delta<1$ is a constant.

Then $\mathcal{F}$ is normal on $D$.

Before we continue to the proof of the main result, let us set some notation.

Throughout, $D$ is a domain in $\mathbb{C}$ and $H_{0}=\left\{w_{0}=0\right\}$ always represents the first coordinate hyperplane. We write $f_{n}(z) \Rightarrow f(z), z \in D$ to indicate that the sequence $\left\{f_{n}\right\}$ converges to $f$ uniformly on compact subsets of $D$ by the Fubini-Study metric on $\mathbb{P}^{N}(\mathbb{C})$. For a holomorphic curve $f(z)$ in $D$, the spherical derivative of $f$ at the point $z$ is still denoted by $f^{\sharp}=\frac{\left|f \wedge f^{\prime}\right|}{\|f\|^{2}}$.

Frequently, given a sequence, $\left\{f_{n}\right\}_{1}^{\infty}$ of maps, we need to extract an appropriate subsequence; and this necessity may recur within a single proof. To avoid the awkwardness of multiple indices, we again denote the extracted subsequence by $\left\{f_{n}\right\}$ (rather than, say, $\left\{f_{n_{k}}\right\}$ ), and signal this operation by "taking a subsequence and renumbering, "or simply "renumbering". The same convention applies to sequence of constants.

\section{Definitions and Notations}

At first, we recall some definitions and notations for $\mathbb{P}^{N}(\mathbb{C})$.

Let $\mathbb{P}^{N}(\mathbb{C})=\mathbb{C}^{N+1} \backslash\{0\} / \sim$ be the $N$-dimensional complex projective space, and for any $x=\left(x_{0}, x_{1}, \cdots, x_{N}\right), y=\left(y_{0}, y_{1}, \cdots, y_{N}\right) \in \mathbb{P}^{N}(\mathbb{C})=\mathbb{C}^{N+1} \backslash\{0\}, x \sim y$ if and only if there exists some $\lambda \in \mathbb{C}$, such that $\left(x_{0}, x_{1}, \cdots, x_{N}\right)=\lambda\left(y_{0}, y_{1}, \cdots, y_{N}\right)$. The equivalence class of $\left(x_{0}, x_{1}, \cdots, x_{N}\right)$ is denoted by $\left[x_{0}: x_{1}: \cdots: x_{N}\right]$ and $\mathbb{P}^{N}(\mathbb{C})=\left\{x=\left[x_{0}: x_{1}: \cdots: x_{N}\right]: x=\left(x_{0}, x_{1}, \cdots, x_{N}\right) \in \mathbb{C}^{N+1} \backslash\{0\}\right\}$.

Let $H_{1}, H_{2}, \cdots, H_{q}$ be hyperplanes in $\mathbb{P}^{N}(\mathbb{C})$ which are given by

$$
H_{\ell}=\left\{x \in \mathbb{P}^{N}(\mathbb{C}):\left\langle x, \alpha_{\ell}\right\rangle=a_{\ell 0} x_{0}+a_{\ell 1} x_{1}+\cdots+a_{\ell N} x_{N}=0\right\}
$$

with nonzero coefficient vectors $\alpha_{\ell}=\left(a_{\ell 0}, a_{\ell 1}, \cdots, a_{\ell N}\right)^{\mathrm{T}}, \ell=1,2, \cdots, q$

Definition 2.1. The hyperplanes $H_{1}, H_{2}, \cdots, H_{q}$ are said to be in general po- 
sition if for any injective maps $\phi:\{0,1,2, \cdots, N\} \rightarrow\{1,2, \cdots, q\}$, the vectors $\alpha_{\phi(0)}, \cdots, \alpha_{\phi(N)}$ are linearly independent.

Second, let $f: D \rightarrow \mathbb{P}^{N}(\mathbb{C})$ be a holomorphic map and $U$ be an open set in $D$. Any holomorphic map $f: U \rightarrow \mathbb{C}^{N+1}$ such that $\mathbb{P}(f(z)) \equiv f(z)$ in $U$ is called a representation of $f$ on $U$, where $\mathbb{P}: \mathbb{C}^{N+1} \backslash\{0\} \rightarrow \mathbb{P}^{N}(\mathbb{C})$ is the standard quotient map.

Definition 2.2. For any open subset $U$ of $D, f=\left(f_{0}, f_{1}, \cdots, f_{N}\right)$ is called to be a reduced representation of $f$ on $U$, if $f_{0}, f_{1}, \cdots, f_{N}$ are holomorphic functions on $U$ without common zeros.

Let $H_{\ell}=\left\{x \in \mathbb{P}^{N}(\mathbb{C}):\langle x, \alpha\rangle=0\right\}$ be a hyperplane, we denote $\|H\|=\|\alpha\|=\max _{0 \leq i \leq N}\left|a_{i}\right|$. Throughout, we only consider normalized hyperplane representations so that $\|H\|=1$

Next, for any reduced representation $f$ of a holomorphic map $f$, we define the holomorphic function.

$$
\langle f(z), H\rangle=\langle f(z), \alpha\rangle=\sum_{i=0}^{N} a_{i} f_{i}(z)
$$

And put

$$
\|f(z)\|=\left\{\sum_{i=0}^{N}\left|f_{i}(z)\right|^{2}\right\}^{1 / 2}
$$

Finally, according to the definition of the derived curves in [7], we have the following definition.

Definition 2.3. Let $f$ be a holomorphic map of $D$ into $\mathbb{P}^{N}(\mathbb{C})$,

$f=\left(f_{0}, f_{1}, \cdots, f_{N}\right)$ be any reduced representation of $f$ on $D$ with $f_{\mu(z)} \neq 0$, $\mu \in\{0,1, \cdots, N\}$. Then

$$
\begin{aligned}
\nabla_{\mu}(f(z))= & {\left[W\left(f_{\mu}, f_{0}\right) / d: \cdots: W\left(f_{\mu}, f_{\mu-1}\right) / d: f_{\mu}^{2} / d: W\left(f_{\mu}, f_{\mu+1}\right) / d\right.} \\
& \left.: \cdots: W\left(f_{\mu}, f_{N}\right) / d\right]
\end{aligned}
$$

is called to be the $\mu$ th derived holomorphic map of $f$, where $d(z)$ be a holomorphic function such that $f_{\mu}^{2} / d$ and $W\left(f_{\mu}, f_{i}\right) / d, \quad(i=0,1, \cdots, N ; i \neq \mu)$ have no common zeros.

Remark 2.1. For simplicity, we also write $\nabla_{\mu} f$ as $\nabla f$, and obviously the definition of $\nabla_{\mu} f$ does not depend on the choice of a reduced represention of $f$.

\section{Preliminary Lemmas}

Before we give the proof of our main theorem, we need the following version of Zalcman's lemma for holomorphic mappings from the domain $\Omega \subseteq \mathbb{C}$ to $\mathbb{P}^{N}(\mathbb{C})$, which was proved by Aladro and Krantz in [8].

Lemma 3.1 (see [8]). Let $\mathcal{F}$ be a family of holomorphic maps of a hyperbolic domain $\Omega \subset \mathbb{C}$ into $\mathbb{P}^{N}(\mathbb{C})$. The family $\mathcal{F}$ is not normal on $\Omega$ if and only if there exist sequences $\left\{f_{n}\right\} \subset \mathcal{F},\left\{z_{n}\right\} \subset \Omega$, with $z_{n} \rightarrow z_{0} \in \Omega,\left\{\rho_{n}\right\}$ and $\rho_{n}>0, \rho_{n} \rightarrow 0$, such that

$$
g_{n}(\zeta):=f_{n}\left(z_{n}+\rho_{n} \zeta\right)
$$


converges uniformly on compact subsets of $\mathbb{C}$ to a nonconstant holomorphic map $g$ of $\mathbb{C}$ into $\mathbb{P}^{N}(\mathbb{C})$.

The degenerate second main theorem in Nevanlina theory shows the following fact

Lemma 3.2 (see ([9], p. 141)). Let $f: \mathbb{C} \rightarrow \mathbb{P}^{N}(\mathbb{C})$ be a holomorphic map, and $H_{1}, \cdots, H_{q}(q \geq 2 N+1)$ be hyperplanes in $\mathbb{P}^{N}(\mathbb{C})$ in general position. If for each $j=1, \cdots, q$, either $f(\mathbb{C})$ is contained in $H_{j}$, or $f(\mathbb{C})$ omits $H_{j}$, then $f$ is constant.

Lemma 3.3 (see ([10], p. 34)). If $f(z)$ and $\varphi_{v}(z)(v=1,2,3)$ are meromorphic functions in the finite plane such that

$$
T\left(r, \varphi_{v}\right)=o\{T(r, f)\}, v=1,2,3
$$

Then we have

$$
\{1-o(1)\} T(r, f)<\sum_{v=1}^{3} N\left(r, \frac{1}{f-\varphi_{v}}\right)+S(r, f)
$$

where $S(r, f)=o\{T(r, f)\}, r \rightarrow \infty$.

Lemma 3.4 (see ([9], p. 124)). Let $f_{0}, f_{1}, \cdots, f_{n+1}$ be nowhere zero entire functions with

$$
f_{0}+f_{1}+\cdots+f_{n+1}=0 .
$$

Consider the partition

$$
\{0,1, \cdots, n+1\}=I_{1} \cup I_{2} \cup \cdots \cup I_{k}
$$

such that $i$ and $j$ are in the same class $I_{\ell}$ if and only if $f_{i}=c_{i j} f_{j}$ for some nonzero constant $c_{i j}$. Then

$$
\sum_{i \in I_{\ell}} f_{i}=0
$$

for any $I_{\ell}$.

Lemma 3.5 (see ([1], p. 5)). Let $g=\left[g_{0}: \cdots: g_{N}\right]: \mathbb{C} \rightarrow \mathbb{P}^{N}(\mathbb{C})$ be a holomorphic curve with finite order and $g_{0}(\zeta) \neq 0$, where $N \geq 2$ is an integer. And let $H_{\ell}=\left\{x \in \mathbb{P}^{N}(\mathbb{C}):\left\langle x, \alpha_{\ell}\right\rangle=0\right\}$ be hyperplanes in $\mathbb{P}^{N}(\mathbb{C})$ located in general position, whose first coefficients $a_{\ell 0}$ are nonzero, for all $\ell=1,2, \cdots, 2 N+1$. Let $g(\zeta)=\left(g_{0}, g_{1}, \cdots, g_{N}\right)(\zeta)$ be any reduced representation of $g$, if we denote

$$
G_{\ell}=a_{\ell 0}+\sum_{i=1}^{N} a_{\ell i} \frac{g_{i}(\zeta)}{g_{0}(\zeta)}, \ell=1,2, \cdots, N+1
$$

and assume that $G_{\ell}(\zeta) \neq 0, \zeta \in \mathbb{C}$ and $G_{\ell}^{\prime}(\zeta) \neq 0, \zeta \in \mathbb{C}$, then we have $g$ is linearly degenerate.

\section{Proof of Theorem 1.1}

Suppose $\mathcal{F}$ is not normal on $D$. Then by Lemma 3.1, there exist points $z_{n} \rightarrow z_{0} \in D$, positive numbers $\rho_{n} \rightarrow 0$ and holomorphic maps $f_{n} \in \mathcal{F}$ such that 


$$
g_{n}(\zeta)=f_{n}\left(z_{n}+\rho_{n} \zeta\right) \Rightarrow g(\zeta), \zeta \in \mathbb{C}
$$

where $g$ is a nonconstant holomorphic map with finite order on $\mathbb{C}$

Let $g(\zeta)=\left(g_{0}, g_{1}, g_{2}, g_{3}, g_{4}\right)(\zeta)$ be the reduced representation of $g$. Since $H_{\ell}, 1 \leq \ell \leq 11$, are in generalposotion, without loss of generality, we may assume that the first coefficients of $H_{1}, H_{2}, H_{3}, H_{4}, H_{5}, H_{6}, H_{7}$ are nonzero.

Case (A). $g$ is linearly nondegenerate.

From the case (A) of theorem LP, we have $G_{\ell}(\zeta) \neq 0$ and $G_{\ell}^{\prime}(\zeta) \neq 0, \ell=1,2,3,4,5,6,7, \zeta \in \mathbb{C}$, then by lemma $3.5, g$ is linearly nondegenerate, a contradiction.

Case (B). $g$ is linearly degenerate.

We can suppose $g_{0}, g_{1}, g_{2}, g_{3}$ are linearly nondegenerate.

Since $G_{\ell} \equiv 0$ or $G_{\ell} \neq 0, \ell=1,2,3,4,5,6,7$, sofrom Lemma 3.5, we have $g_{0} \neq 0$ and $g$ is a holomorphic map.

Then $G_{\ell}$ is holomorphic in $\mathbb{C}$. And we have $\rho_{G_{\ell}} \leq 1,1 \leq \ell \leq 7$.

Then $G_{\ell}=a_{\ell 0}+a_{\ell 1} \frac{g_{1}}{g_{0}}+a_{\ell 2} \frac{g_{2}}{g_{0}}+a_{\ell 3} \frac{g_{3}}{g_{0}}+a_{\ell 4} \frac{g_{4}}{g_{0}}=B_{\ell} \mathrm{e}^{A_{\ell} \zeta}, 1 \leq \ell \leq 7$. Specially, when $G_{\ell} \equiv 0, B_{\ell}=0$.

$\forall j_{1}, j_{2}, j_{3}, j_{4} \in\{1,2,3,4,5,6,7\}$, without loss of generality, $j_{1}=1, j_{2}=2, j_{3}=3, j_{4}=4, j_{5}=5$.

Let $k_{1} B_{1} \mathrm{e}^{A_{1} \zeta}+k_{2} B_{2} \mathrm{e}^{A_{2} \zeta}+k_{3} B_{3} \mathrm{e}^{A_{3} \zeta}+k_{4} B_{4} \mathrm{e}^{A_{4} \zeta}+k_{5} B_{5} \mathrm{e}^{A_{5} \zeta}=0, \quad k_{1}, k_{2}, k_{3}, k_{4}$ and $k_{5}$ are constants.

Since $1, \frac{g_{1}}{g_{0}}, \frac{g_{2}}{g_{0}}, \frac{g_{3}}{g_{0}}, \frac{g_{4}}{g_{0}}$ are linearly degenerate, then there exist a nonzero vector $\left(b_{1}, b_{2}, b_{3}, b_{4}, b_{5}\right)^{\mathrm{T}}$ such that

$$
\left(1, \frac{g_{1}}{g_{0}}, \frac{g_{2}}{g_{0}}, \frac{g_{3}}{g_{0}}, \frac{g_{4}}{g_{0}}\right)\left(\begin{array}{l}
b_{1} \\
b_{2} \\
b_{3} \\
b_{4} \\
b_{5}
\end{array}\right)=0
$$

Let

$$
A=\left(\begin{array}{lllll}
a_{10} & a_{20} & a_{30} & a_{40} & a_{50} \\
a_{11} & a_{21} & a_{31} & a_{41} & a_{51} \\
a_{12} & a_{22} & a_{32} & a_{42} & a_{52} \\
a_{13} & a_{23} & a_{33} & a_{43} & a_{53} \\
a_{14} & a_{24} & a_{34} & a_{44} & a_{54}
\end{array}\right)
$$

Since $|A| \neq 0$, then let $\left(k_{1}, k_{2}, k_{3}, k_{4}, k_{5}\right)^{\mathrm{T}}=A^{-1}\left(b_{1}, b_{2}, b_{3}, b_{4}, b_{5}\right)^{\mathrm{T}}$. It is a nonzero vector. Then $B_{1} \mathrm{e}^{A_{1} \zeta}, B_{2} \mathrm{e}^{A_{2} \zeta}, B_{3} \mathrm{e}^{A_{3} \zeta}, B_{4} \mathrm{e}^{A_{4} \zeta}, B_{5} \mathrm{e}^{A_{5} \zeta}$ are linearly degenerate.

Claim. There exist an injective map $\sigma:\{1,2,3,4\} \rightarrow\{1,2,3,4,5\}$, such that $B_{\sigma(1)} \mathrm{e}^{A_{\sigma(1)} \zeta}, B_{\sigma(2)} \mathrm{e}^{A_{\sigma(2)} \zeta}, B_{\sigma(3)} \mathrm{e}^{A_{\sigma(3)} \zeta}, B_{\sigma(4)} \mathrm{e}^{A_{\sigma(4)} \zeta}$ are linearly nondegenerate.

Proof of claim. Without loss of generality, we may assume that there exists some constants $\ell_{1}, \ell_{2}, \ell_{3}, \ell_{4}$, such that 
$B_{5} \mathrm{e}^{A_{5} \zeta}=\ell_{1} B_{1} \mathrm{e}^{A_{1} \zeta}+\ell_{2} B_{2} \mathrm{e}^{A_{2} \zeta}+\ell_{3} B_{3} \mathrm{e}^{A_{3} \zeta}+\ell_{4} B_{4} \mathrm{e}^{A_{4} \zeta}$

Since

$$
\left(B_{1} \mathrm{e}^{A_{1} \zeta}, B_{2} \mathrm{e}^{A_{2} \zeta}, B_{3} \mathrm{e}^{A_{3} \zeta}, B_{4} \mathrm{e}^{A_{4} \zeta}, B_{5} \mathrm{e}^{A_{5} \zeta}\right)=\left(1, \frac{g_{1}}{g_{0}}, \frac{g_{2}}{g_{0}}, \frac{g_{3}}{g_{0}}, \frac{g_{4}}{g_{0}}\right) A
$$

Then $\left(1, \frac{g_{1}}{g_{0}}, \frac{g_{2}}{g_{0}}, \frac{g_{3}}{g_{0}}, \frac{g_{4}}{g_{0}}\right)=\left(B_{1} \mathrm{e}^{A_{1} \zeta}, B_{2} \mathrm{e}^{A_{2} \zeta}, B_{3} \mathrm{e}^{A_{3} \zeta}, B_{4} \mathrm{e}^{A_{4} \zeta}, B_{5} \mathrm{e}^{A_{5} \zeta}\right) A^{-1}$

Then we can let

$$
\frac{g_{j}}{g_{0}}=\sum_{i=1}^{4} c_{i j} B_{i} \mathrm{e}^{A_{i} \zeta}, j=0,1,2,3
$$

Let

$$
C=\left(\begin{array}{llll}
c_{10} & c_{11} & c_{12} & c_{13} \\
c_{20} & c_{21} & c_{22} & c_{23} \\
c_{30} & c_{31} & c_{32} & c_{33} \\
c_{40} & c_{41} & c_{42} & c_{43}
\end{array}\right)
$$

If $r(C) \leq 3$, which implies the equation set

$$
C\left(\begin{array}{l}
q_{0} \\
q_{1} \\
q_{2} \\
q_{3}
\end{array}\right)=0
$$

Have untrivial solution, then there exist some constants $q_{0}, q_{1}, q_{2}, q_{3}$, which are not identical to zero, such that

$$
\left(B_{1} \mathrm{e}^{A_{1} \zeta}, B_{2} \mathrm{e}^{A_{2} \zeta}, B_{3} \mathrm{e}^{A_{3} \zeta}, B_{4} \mathrm{e}^{A_{4} \zeta}\right) C\left(\begin{array}{c}
q_{0} \\
q_{1} \\
q_{2} \\
q_{3}
\end{array}\right)=0
$$

i.e.

$$
\left(1, \frac{g_{1}}{g_{0}}, \frac{g_{2}}{g_{0}}, \frac{g_{3}}{g_{0}}\right)\left(\begin{array}{c}
q_{0} \\
q_{1} \\
q_{2} \\
q_{3}
\end{array}\right)=0
$$

Then $1, \frac{g_{1}}{g_{0}}, \frac{g_{2}}{g_{0}}, \frac{g_{3}}{g_{0}}$ are linear degenerate, a contradiction and $r(C)=3$.

If $B_{1} \mathrm{e}^{A_{1} \zeta}, B_{2} \mathrm{e}^{A_{2} \zeta}, B_{3} \mathrm{e}^{A_{3} \zeta}, B_{4} \mathrm{e}^{A_{4} \zeta}$ are linearly degenerate, then there exists some constants $c_{0}, c_{1}, c_{2}, C_{3}$, which are not identical to zero, such that

$$
\left(B_{1} \mathrm{e}^{A_{1} \zeta}, B_{2} \mathrm{e}^{A_{2} \zeta}, B_{3} \mathrm{e}^{A_{3} \zeta}, B_{4} \mathrm{e}^{A_{4} \zeta}\right)\left(\begin{array}{c}
c_{0} \\
c_{1} \\
c_{2} \\
c_{3}
\end{array}\right)=0
$$

By $\left({ }^{*}\right)$, we have 


$$
\left(1, \frac{g_{1}}{g_{0}}, \frac{g_{2}}{g_{0}}, \frac{g_{3}}{g_{0}}\right) C^{-1}\left(\begin{array}{c}
c_{0} \\
c_{1} \\
c_{2} \\
c_{3}
\end{array}\right)=0
$$

which implies $1, \frac{g_{1}}{g_{0}}, \frac{g_{2}}{g_{0}}, \frac{g_{3}}{g_{0}}$ are linearly degenerate, a contradiction. The claim is proven.

Then $B_{1} \mathrm{e}^{A_{1} \zeta}, B_{2} \mathrm{e}^{A_{2} \zeta}, B_{3} \mathrm{e}^{A_{3} \zeta}, B_{4} \mathrm{e}^{A_{4} \zeta}$ are linearly nondegenerate, which implies $A_{1}, A_{2}, A_{3}, A_{4}$ are different from easy other.

Since $H_{\ell}, 1 \leq \ell \leq 6$ be hyperplanes in $\mathbb{P}^{4}(\mathbb{C})$ located in general position.

Then every five exist four differences in $A_{1}, A_{2}, A_{3}, A_{4}, A_{5}, A_{6}$

If there exist $l \in\{1,2,3,4,5,6\}$, such that $B_{l}=0$, without loss of generality, we can let $B_{6}=0$, so $B_{i} \neq 0, i=1,2,3,4,5$

Since $B_{1} \mathrm{e}^{A_{1} \zeta}, B_{2} \mathrm{e}^{A_{2} \zeta}, B_{3} \mathrm{e}^{A_{3} \zeta}, B_{4} \mathrm{e}^{A_{4} \zeta}, B_{5} \mathrm{e}^{A_{5} \zeta}$ are linearly degenerate. Without loss of generality, let $A_{1}=A_{2}$

But obviously, there are not exists four linearly nondegenerate in $B_{1} \mathrm{e}^{A_{1} \zeta}, B_{2} \mathrm{e}^{A_{2} \zeta}, B_{3} \mathrm{e}^{A_{3} \zeta}, B_{4} \mathrm{e}^{A_{4} \zeta}, B_{6} \mathrm{e}^{A_{6} \zeta}$, a contradiction. So $B_{l} \neq 0, l=1,2,3,4,5,6$

Since $\forall j_{1}, j_{2}, j_{3}, j_{4}, j_{5} \in\{1,2,3,4,5,6\}$, $B_{j_{1}} \mathrm{e}^{A_{j_{1}} \zeta}, B_{j_{2}} \mathrm{e}^{A_{j_{2}} \zeta}, B_{j_{3}} \mathrm{e}^{A_{j_{3}} \zeta}, B_{j_{4}} \mathrm{e}^{A_{j_{4}} \zeta}, B_{j_{5}} \mathrm{e}^{A_{j_{5}} \zeta}$ are linearly degenerate, which mean that there exists two identical in $A_{j_{1}}, A_{j_{2}}, A_{j_{3}}, A_{j_{4}}, A_{j_{5}}$

Then every five exists two identical in $A_{1}, A_{2}, A_{3}, A_{4}, A_{5}, A_{6}$.

If $A_{1}, A_{2}, A_{3}, A_{4}$ are differences, then $A_{5}$ equals one of $A_{1}, A_{2}, A_{3}, A_{4}$, let $A_{5}=A_{1}$. Similarly, $A_{6}$ equals one of $A_{1}, A_{2}, A_{3}, A_{4}$, let $A_{6}=A_{2}$.

Then it is impossible to exist four differences in $A_{1}, A_{2}, A_{4}, A_{5}, A_{6}$, a contradiction, so $g_{0}, g_{1}, g_{2}, g_{3}$ are linearly degenerate.

If $g_{0}, g_{1}, g_{2}$ are linearly nondegenerate, then $r\left(g_{0}, g_{1}, g_{2}, g_{3}, g_{4}\right)=3$

Since every four linearly independent in $\alpha_{1}, \alpha_{2}, \alpha_{3}, \alpha_{4}, \alpha_{5}, \alpha_{6}, \alpha_{7}$. Let

$B=\left[\alpha_{1}, \alpha_{2}, \alpha_{3}, \alpha_{4}\right], \quad r(B)=4$, then

$r\left(\left(g_{0}, g_{1}, g_{2}, g_{3}, g_{4}\right) B\right)=r\left(g_{0}, g_{1}, g_{2}, g_{3}, g_{4}\right)=3$

Since

$$
\left(1, \frac{g_{1}}{g_{0}}, \frac{g_{2}}{g_{0}}, \frac{g_{3}}{g_{0}}, \frac{g_{4}}{g_{0}}\right) B=\left(B_{1} \mathrm{e}^{A_{1} \zeta}, B_{2} \mathrm{e}^{A_{2} \zeta}, B_{3} \mathrm{e}^{A_{3} \zeta}, B_{4} \mathrm{e}^{A_{4} \zeta}\right)
$$

then exist $i \neq j$, such that $A_{i}=A_{j}, \quad i, j \in\{1,2,3,4\}$

If $A_{1}, A_{2}, A_{3}$ are different, so $A_{4}, A_{5}, A_{6}, A_{7}$ equal one of $A_{1}, A_{2}, A_{3}$, let $A_{4}=A_{1}, A_{5}=A_{2}, A_{6}=A_{3}, A_{7}=A_{1}$, then it is obviously that there not exist three differences in $A_{1}, A_{4}, A_{5}, A_{6}, A_{7}$, a contradiction.

So $g_{0}, g_{1}, g_{2}$ are linearly degenerate.

So there exist constants $p_{0}, p_{1}, p_{2}$ which are not identical to zero, such that $p_{0} g_{0}+p_{1} g_{1}+p_{2} g_{2}=0$

1) $p_{2} \neq 0$, then $g_{2}=-\frac{p_{0}}{p_{2}} g_{0}-\frac{p_{1}}{p_{2}} g_{1}$ and $g_{2}, g_{3}, g_{4}$ can be linear representation by $g_{0}, g_{1}$. 
So there exist constants $k_{1}, k_{2}, l_{1}, l_{2}$, such that $g_{2}=p_{1} g_{0}+p_{2} g_{1}$, $g_{3}=k_{0} g_{0}+k_{1} g_{1}+k_{2} g_{2}, \quad g_{4}=l_{0} g_{0}+l_{1} g_{1}+l_{2} g_{2}+l_{3} g_{3}$

Then

$$
\begin{aligned}
G_{\ell}= & a_{\ell 0}+a_{\ell 1} \frac{g_{1}}{g_{0}}+a_{\ell 2} \frac{g_{2}}{g_{0}}+a_{\ell 3} \frac{g_{3}}{g_{0}}+a_{\ell 4} \frac{g_{4}}{g_{0}} \\
= & a_{\ell 0}+p_{0} a_{l_{2}}+a_{l_{3}}\left(k_{0}+k_{2} p_{0}\right)+a_{l_{4}}\left(l_{0}+l_{2} p_{0}+l_{3} k_{0}+l_{3} k_{2} p_{0}\right) \\
& +\left[a_{l_{1}}+a_{l_{2}} p_{1}+a_{l_{3}}\left(k_{1}+k_{2} p_{1}\right)+a_{l_{4}}\left(l_{2} p_{1}+l_{1}+l_{3}\left(k_{1}+k_{2} p_{1}\right)\right)\right] \frac{g_{1}}{g_{0}}
\end{aligned}
$$

Since $\alpha_{1}, \alpha_{2}, \alpha_{3}, \alpha_{4}, \alpha_{5}$ are linearly independent, then exist $\ell \in\{1,2,3,4,5\}$, such that $a_{l_{1}}+a_{l_{2}} p_{1}+a_{l_{3}}\left(k_{1}+k_{2} p_{1}\right)+a_{l_{4}}\left(l_{2} p_{1}+l_{1}+l_{3}\left(k_{1}+k_{2} p_{1}\right)\right) \neq 0$

If for such $\ell, G_{\ell}^{\prime} \equiv 0$, which implies that $\frac{g_{1}}{g_{0}} \equiv C$, a contradiction. Then, we have $G_{\ell}^{\prime} \neq 0$ and $\left(\frac{g_{1}}{g_{0}}\right)^{\prime} \neq 0$.

From lemma 3.2, without loss of generality, we may assume the first coefficient of $H_{8}$ is nonzero, i.e. $a_{80} \neq 0$. If the first coefficient of $H_{9}$ are still nonzero, i.e. $a_{90} \neq 0$, we have g omits $H_{9}$ or $g(\mathbb{C})$ is contained in $H_{9}$.

Since $\alpha_{8}=\left(0, a_{81}, a_{82}, a_{83}, a_{84}\right)^{\mathrm{T}}$, we have all zeros of $\left\langle g, \alpha_{8}\right\rangle$ are multiple. Thus, all zeros of

$$
\begin{aligned}
G_{8}= & p_{0} a_{82}+a_{83}\left(k_{0}+k_{2} p_{0}\right)+a_{84}\left(l_{0}+l_{2} p_{0}+l_{3} k_{0}+l_{3} k_{2} p_{0}\right) \\
& +\left[a_{81}+a_{82} p_{1}+a_{83}\left(k_{1}+k_{2} p_{1}\right)+a_{84}\left(l_{2} p_{1}+l_{1}+l_{3}\left(k_{1}+k_{2} p_{1}\right)\right)\right] \frac{g_{1}}{g_{0}}
\end{aligned}
$$

are multiple.

If for all $\zeta \in \mathbb{C},\left\langle g, \alpha_{8}\right\rangle \neq 0$ or $\equiv 0$. By lemma 3.2, we have $g$ is a constant curve, a contradiction.

Then, there exists some $\zeta_{0} \in \mathbb{C}$, such that $\left\langle g, \alpha_{8}\right\rangle\left(\zeta_{0}\right)=0$ and $\left\langle g, \alpha_{8}\right\rangle(\zeta) \neq 0$. This implies that

$$
\left.\left[a_{81}+a_{82} p_{1}+a_{83}\left(k_{1}+k_{2} p_{1}\right)+a_{84}\left(l_{2} p_{1}+l_{1}+l_{3}\left(k_{1}+k_{2} p_{1}\right)\right)\right]\left(\frac{g_{1}}{g_{0}}\right)^{\prime}\right|_{\zeta_{0}}=0,
$$

a contradiction.

Thus, the first coefficient of $H_{9}$ are still zero, i.e. $a_{90}=0$. Similarly, we have all zeros of $\left\langle g, \alpha_{\ell}\right\rangle$ are multiple, we have all zeros of

$$
\begin{aligned}
G_{\ell}= & p_{0} a_{l_{2}}+a_{l_{3}}\left(k_{0}+k_{2} p_{0}\right)+a_{l_{4}}\left(l_{0}+l_{2} p_{0}+l_{3} k_{0}+l_{3} k_{2} p_{0}\right) \\
& +\left[a_{l_{1}}+a_{l_{2}} p_{1}+a_{l_{3}}\left(k_{1}+k_{2} p_{1}\right)+a_{l_{4}}\left(l_{2} p_{1}+l_{1}+l_{3}\left(k_{1}+k_{2} p_{1}\right)\right)\right] \frac{g_{1}}{g_{0}}, \ell=8,9
\end{aligned}
$$

are multiple.

If for all $\zeta \in \mathbb{C},\left\langle g, \alpha_{\ell}\right\rangle \neq 0$ or $\equiv 0, \ell=8,9$. By lemma 3.2, we have $g$ is a constant curve, a contradiction.

Then, there exist some $\zeta_{0} \in \mathbb{C}, \ell=8$ or 9 , such that $\left\langle g, \alpha_{\ell}\right\rangle\left(\zeta_{0}\right)=0$ and 
$\left\langle g, \alpha_{\ell}\right\rangle(\zeta) \not \equiv 0$. This implies that

$$
\left[a_{l_{1}}+a_{l_{2}} p_{1}+a_{l_{3}}\left(k_{1}+k_{2} p_{1}\right)+a_{l_{4}}\left(l_{2} p_{1}+l_{1}+l_{3}\left(k_{1}+k_{2} p_{1}\right)\right)\right]\left(\frac{g_{1}}{g_{0}}\right)_{\zeta_{0}}^{\prime}=0,
$$

a contradiction.

2) $p_{2}=0$, then $p_{0}, p_{1}$ are not identical to zero.

a) $p_{0} \neq 0$, a contradiction.

b) $p_{0}=0$, then $p_{1} \neq 0$, which implies $g_{1} \equiv 0$, then $\frac{g_{1}}{g_{0}} \equiv 0$, a contradiction.

Thus, $\mathcal{F}$ is normal on $D$. The proof of Theorem 1.1 is finished

So in a word, we can make a conclusion as follows:

Theorem 1.1. Let $\mathcal{F}$ be a family of holomorphic maps of a domain $D \subset \mathbb{C}$ to $\mathbb{P}^{4}(\mathbb{C})$. Let $H_{0}=\left\{w_{0}=0\right\} \quad$ and $H_{l}=\left\{x \in \mathbb{P}^{4}(\mathbb{C}):\left\langle x, \alpha_{l}\right\rangle=0\right\} \neq H_{0}$ be hyperplanes in $\mathbb{P}^{4}(\mathbb{C})$ located in general position, where

$\alpha_{l}=\left(\alpha_{l_{0}}, \alpha_{l_{1}}, \alpha_{l_{2}}, \alpha_{l_{3}}, \alpha_{l_{4}}\right)^{\mathrm{T}}, l=1,2,3, \cdots, 11$

Assume the following conditions hold for every $f \in \mathcal{F}$ :

1) $f(z) \in H_{l}$ if and only if $\nabla f \in H_{l}, l=1,2,3, \cdots, 11$

2) If $f(z) \in \bigcup_{l=1}^{11} H_{l}$, then $\frac{\left\langle f(z), H_{0}\right\rangle \mid}{\|f\|\left\|H_{0}\right\|} \geq \delta$, where $0<\delta<1$ is a constant.

Then $\mathcal{F}$ is normal on $D$.

\section{Conflicts of Interest}

The author declares no conflicts of interest regarding the publication of this paper.

\section{References}

[1] Liu, X.J. and Pang, X.C. (2020) Shared Hyperplanes and Normal Families of Holomorphic Curves. International Journal of Mathematics, 31, Article ID: 2050037. https://doi.org/10.1142/S0129167X20500378

[2] Zalcman, L. (1975) A Heuritic Principle in Complex Function Theory. The American Mathematical Monthly, 82, 813-817. https://doi.org/10.1080/00029890.1975.11993942

[3] Zalcman, L. (1998) Normal Families: New Perspectives. Bulletin of the American Mathematical Society, 35, 215-230. https://doi.org/10.1090/S0273-0979-98-00755-1

[4] Chen, Z.H. and Yan, Q.M. (2009) Uniqueness Theorem of Meromorphic Mappings into $\mathbb{P}^{N}(\mathbb{C})$ Sharing $2 \mathrm{~N}+3$ Hyperplanes Regardless of Multiplicities. International Journal of Mathematics, 20, 717-726. https://doi.org/10.1142/S0129167X09005492

[5] Yang, L., Fang, C.Y. and Pang, X.C. (2014) Normal Families of Holomorphic Mappings into Complex Projective Space Concerning Shared Hyperplanes. Pacific Journal of Mathematics, 272, 245-256. https://doi.org/10.2140/pjm.2014.272.245

[6] Liu, X.J., Pang, X.C. and Yang, J.H. (2017) A Criterion of Normality Concerning Shared Hyperplanes. Chin. Chinese Annals of Mathematics, Series B, 38, 1-6.

[7] Ye, Y.S. Yang, L. and Pang, X.C. (2015) An Extension of Schwick's Theorem for 
Normal Families. Annales Polonici Mathematici, 115, 23-31.

https://doi.org/10.4064/ap115-1-2

[8] Aladroand, G. and Krantz, S.G. (1991) A Criterion for Normality in $\mathbb{C}^{n}$. Journal of Mathematical Analysis and Applications, 161, 1-8.

https://doi.org/10.1016/0022-247X(91)90356-5

[9] Ru, M. (2001) Nevanlinna Theory and Its Relation to Diophantine Approximation. World Scientific, Singapore. https://doi.org/10.1142/4508

[10] Yang, L. (1993) Value Distribution Theory. Springer-Verlag, Berlin. 